\title{
Information Management for Life Cycle Assessment using Smart Agents
}

G. Seliger, T. Keil, D. Krützfeldt, K. Müller, H. Perlewitz Technical University of Berlin

Institute for Machine Tools and Factory Management

Department of Assembly Technology and Factory Management

Pascalstraße 8-9, 10587 Berlin, Germany

Phone: +49-30-314-24457, Fax: +49-30-314-22759

e-mail: Holger.Perlewitz@iwf-mt.tu-berlin.de

\begin{abstract}
An environmental-friendly and effective cycle economy calls for an open information connection concerning the designers, the recyclers and the manufacturers of recycling techniques. Disposal and recycling information has to be available for the design, including the product assessment. The recycler and the manufacturer of recycling techniques need general conditions and product information from the design. The requirements for the information system are derived from these needs. The concept designates a standardised data model. A federalised data base system with appropriate transformations is suggested for the data organisation.
\end{abstract}

Keywords

Integrated Product and Process Planning, Recycling, Knowledge Acquisition 


\section{SUCCESS BY INFORMATION}

Cycle economy as a paradigm for industry in the 21 st century aims at economically and responsibly dealing with our limited resources. In order to reach economical and environmental-friendly cycles the requirements of recycling already have to be taken into consideration during product design. Disassembly and recycling companies have to be efficiently organised and have to possess special technology that fulfils the special quality and quantity requirements concerning work material and components during the manufacturing process. There is a requirement of cooperation between the manufacturer, the user and the developer of recycling technique. The challenge for the management of cycle economy companies' lies in an open and continuous flow of information between the firms.

\section{The Designer in the Cycle Economy}

Life cycle oriented product design shall lead to a maximum of usage while minimising the economical, ecological and social efforts during product life. Requirements of different stages in the product life cycle compete when designing a product. Using life cycle assessments design alternatives can be compared and selected. The assessment of the recycling and the disposal stage includes some special features. When designing products the designer has to face the problem that he can not fix the kind and dimension of recovery exactly. His decisions about which components have to be reused or which materials utilised strongly depend on design trends, anticipated state of the art of recycling technologies and future economical, ecological and legal conditions.

There are two challenges assessing the recycling-suitability of a product within the frame of a life cycle assessment:

- There usually exist a great number of different recycling-processes and usagepossibilities to be taken into account.

- The settings of economical, ecological and legal conditions in the future influencing decisions regarding recycling-processes and usage-possibilities are hard to predict due to the long time span between design and recycling.

During the assessment of the recycling suitability all recovery possibilities of a product, its components and materials are modelled in a recovery graph and subsequently evaluated (Figure 1) (Zussman et al. 1994). 


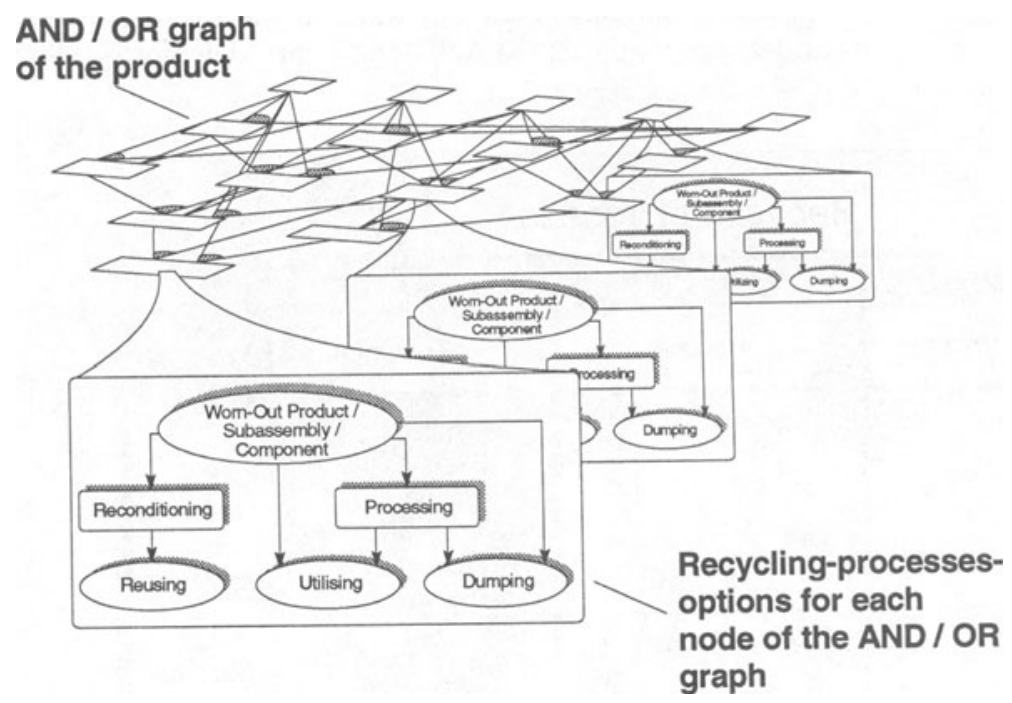

\section{Figure 1 Recovery Graph}

In order to do so all disassembly operations required and all consecutive recycling possibilities are determined. An algorithm evaluates a favourable recovery plan. This plan includes the necessary disassembly operations, their order and the following utilisation or disposal. Therefore the designer needs comparable information on disassembly and recycling procedures. The future development of recycling processes requires updated process information concerning the life cycle of a product. The producer can adapt his recycling strategies to the new conditions and act in time. Actions could be, for example, the contraction of co-operating disassemblers and recyclers or the introduction of a bonus system for returned products in case of increasing gains due to recycling.

Besides information on recycling techniques, the designer can also receive references for the improvement of his work through a co-operation with recycling companies. This requires an evaluation of the recycling results.

\section{The Developer of Recycling Techniques for Cycle Economy}

In addition of conventional ways for waste disposal (dumping and burning), nowadays, there is a development of various recycling methods especially concerning the treatment of plastics and electronic parts. The commencements for the development are:

- future products for disposal

- economical, ecological and legal general conditions

- desired parts and work materials from the recycling process

The developer of recycling techniques has to arrange his facility according to the input which is defined by the designer and to the output which is expected by the recycler. An automatic assignment of recycling alternatives compares the recycling suitability of a product. 
The recycling process is separated into three elements which cover a product from its end of usage until its utilisation (Figure 2) (Nickel 1996, Löhr, Melchiorre 1995, VDI 2243).

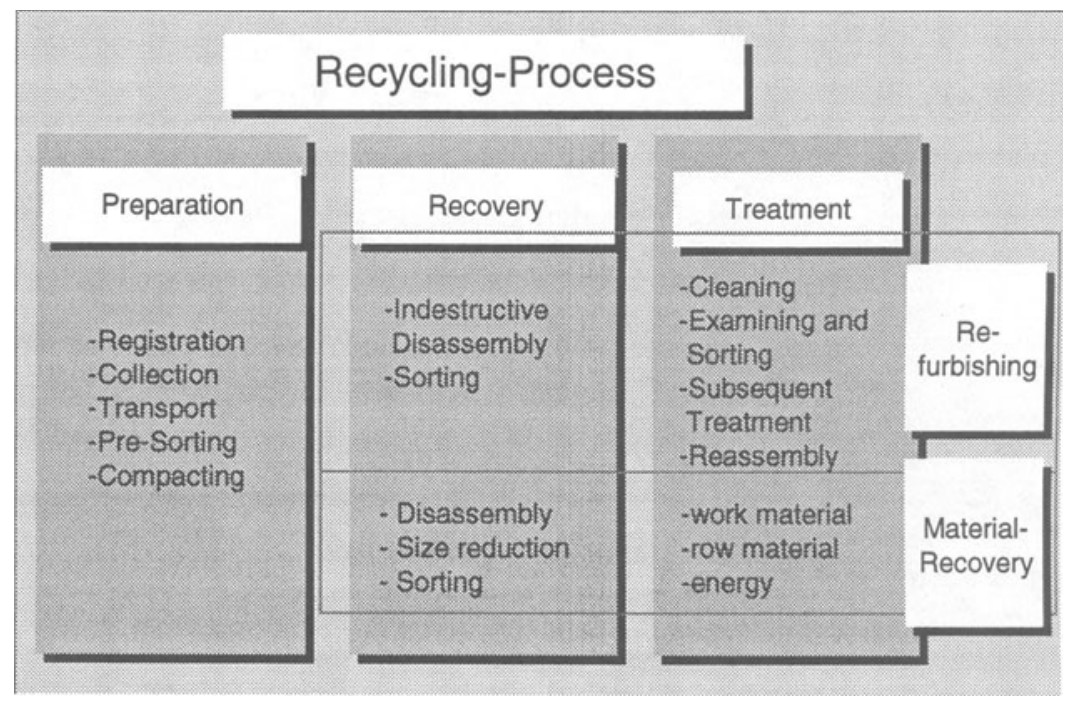

Figure 2 Systematic of recycling-processes

The refurbishing process includes the recovery and the treatment on product basis, whereas the material recovery process treats and recovers the products as materials. The different recycling methods are classified through these systematic.

Each procedure is characterised through:

- access restrictions related to material and shape

- process features: fixed parameters like depreciation and variable parameters like flow and selectivity

- output parameters as a function of input parameters e.g. requirement of energy

\section{The Recycler in the Cycle Economy}

Recycling is proposed to avert pollution and danger from a used product and to benefit after its usage. Frequently, a simple disposal of the product is cheaper than recycling because disassembly, refurbishing, material recovery and the related processes are too expensive.

Some reasons are:

- missing product information which could include inquiries on product condition

- 'backstream amounts' that are difficult to estimate

- lots of product varieties following different disassembly methods

The economical organisation of cycles is supported by the kind, amount, structure and the condition of a product as well as by ensured access during its usage. While 
the recovery graph is available during the design stage, product information concerning the usage can for example be registered by information tools. Nowadays, diagnostic systems are already used in cars or machine tools continuously supplying information about product conditions. Another operation area of diagnostic systems is the registration of product conditions during service and maintenance. Usage and utilisation possibilities can be reliably determined. Using the information from the recycling technique developer, the recycler is able to chose the most suitable process that changes the existing input into the claimed output.

\section{Requirements for an Information Technological Infrastructure}

Figure 3 shows the information flow between designers, recyclers and developers of recycling technologies

To support the co-operation in product life cycle an agile information system is required. These have to fulfil following functions ( Song et al 1995):

1. Provide consistent information to dispersed partners. Data constraints representation and maintenance are important such as for the life cycle assessment and translation of the recovery plan.

2. Provide multiple information views to partners. Partners are from various domains and perform various tasks in the cycle economy. Representation, manipulation and updating of shared information.

3. Provide necessary information security to partners. Co-operations between partners are dynamic. This is important to competing companies that co-operate in the cycle economy. A partner may have limits in retrieving or updating another partner's data.

4. Provide flexible schemes to reflect company-specific policies and procedures so that partners' heterogeneity and autonomy are unaffected. Different data bases and data structures are existent and have to integrate into the information system.

5. Search in distributed data sources and the establishment of transfer concerning the hard and software.

6. The entire system does not depend on the interplay of all participants 


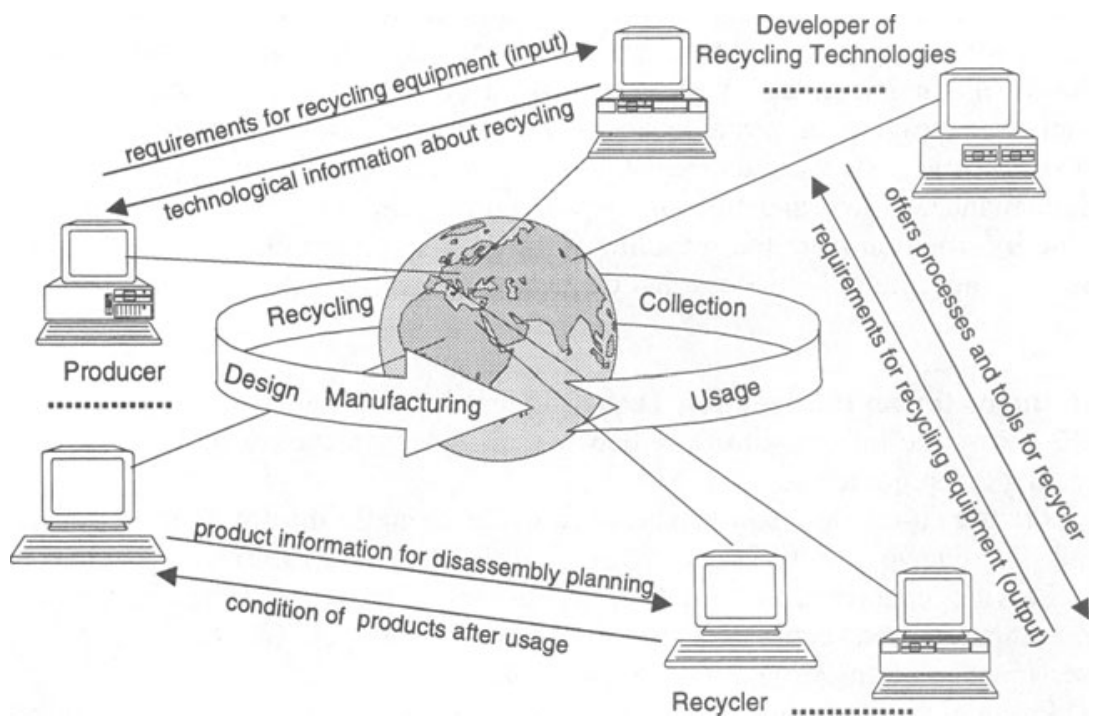

Figure 3 Information flow

In the following, possibilities for data management, data distribution and data search are checked for suitability.

\section{CONCEPTS FOR THE INFORMATION TECHNOLOGICAL INFRASTRUCTURE}

\section{Data management and distribution}

Data bases are used for the management and use of large data amounts. Important aspects are the data organisation as well as the separation of users' data. Users are connected to the system according to defined interfaces. The data base system contains the actual data base and the data base management. The management system supports functions for the maintenance, query and processing of the data.

Data structures and models are used for the logical description of data and for their relations. The relational model is frequently used as a standard in industrial applications. New systems use object oriented approaches for data modelling. Advantages are the application of object classes as well as concepts for inheritance and encapsulation.

Data can be kept in an agile data base system. The centralisation can refer to the local data storage or to the organised data management. In complex systems, information are often kept decentral. Companies benefit from this decentralisation. Problems are the consistency, the redundancy and the data security.

The decentralisation refers to the distribution of data and the data base access. A separation into centralised, distributed and federalised systems is made. 
- Centralised systems are characterised by central data and data storage. The communication causes an additional administration management.

- Distributed systems cover logically connected data bases. The data base structure can be kept either centralised in a node, redundant in each computer or as local parts referring to the entire model.

- Totally independent data base systems are connected by federalised data base systems. This corresponds to an accumulation of autonomous but cooperating data bases. The system does not have a centralised control mechanism ( Dreer et al 1995 ).

According to these points, the federalised data base system is the most suitable one to fulfil the requirements.

The distribution of data is accomplished by client/server applications with varying functions. Using file servers, resources can be accessed by application groups. Data base servers support distributed data base queries via SQL. The separation of the data base and the distributed access increase the system performance. The data manipulation, data independence and data integrity is still secured. These functions are important for consistent product and process data. Frequently developed systems fulfil these functions with distributed objects. Common standards of object definition and access as COBRA, COM or HLA support the platform independence and the communication between different systems (Orfali et al 1996).

Servers are frequently the most suitable variant to develop a general concept. However, they will be replaced by object oriented bases in the future.

\section{Data search}

Simple query and analyse tools build the interface to data base systems. They enable the search in structured data systems. Further functions for the administration of multidimensional data models are realised through Online Analytical Processes (OLAP) Tools.

Data mining includes the possibility of search in highly chaotic information sources via Internet. Unexpected structures can be discovered. The output is accomplished according to the IF-Then rules. As an example, the search for disassembly and automobile could lead to the result: a) the processes and b) the statement: IF disassembly; and automobile; THEN 50\% done by the manufacturer.' Further developments are personal information agents. They do not only supply results out of chaotic data structures, but also give decision support. Using these agents, the most cost-effective process can be selected. There is also the possibility of a material choice according to the selected process.

Search efficiency and user efficiency are important characteristics to select a suited system. Information agents offer far-reaching possibilities for the effective information search. 


\section{FEDERATION IN LIFE CYCLE ECONOMY}

\section{Data modelling}

The starting points for data management, data distribution and data search in distributed systems are exchangeable contents. The unified data meta model is the amount of all relevant data that is to be distributed. The structure can be centrally administrated or composed of single local submodels. Figure 4 shows the example of the life cycle assessment where the relevant modules of a data base, concerning the disassembly and recycling, are introduced.

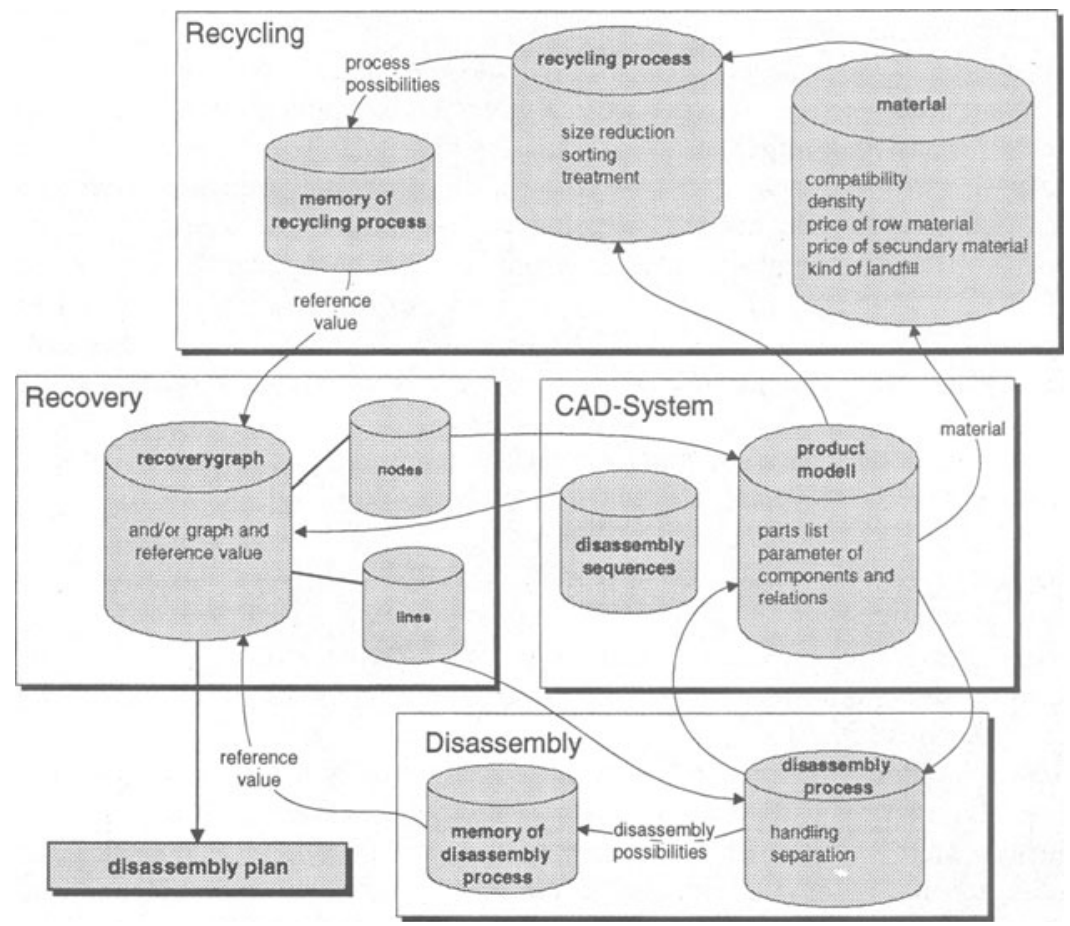

Figure 4 Data model for life cycle assessment

Figure 5 shows the algorithm for the assignments of recycling processes. 


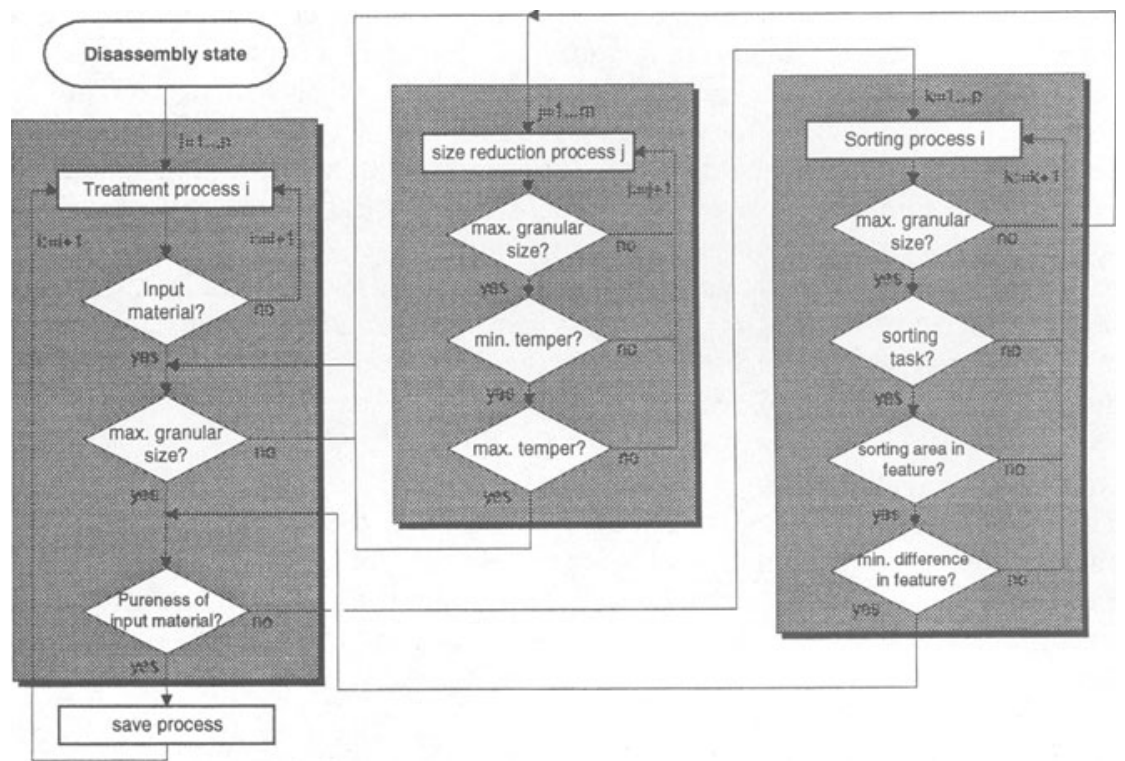

\section{Figure 5 Assignment algorithm}

The unified data meta model of recycling processes is arranged in three categories: process characteristics, input and output. Figure 6 shows such a model treating the example of sorting methods. Within the process methods, further arrangements, concerning the process technical functions, were made. Regarding these points, the sorting procedure is subdivided into particle sorting and material sorting.

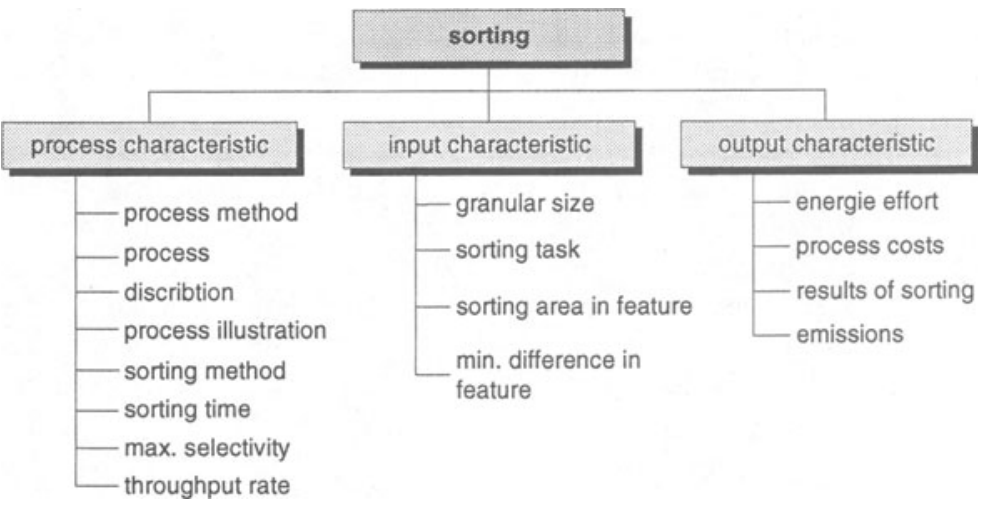

Figure 6

Template of sorting processes 
The sorting method describes the characteristics e.g. density, magnetism and electrostatic charge which are responsible for the sorting of material. The selectivity quantifies the recoverable material out of the mixture. The input characteristic specify the restrictions. These restrictions are: the sorting task that describes the sortable materials, the sorting area within the sorting features, for example the minimum and maximum density and the minimal difference in the sorting characteristic such as the permissible density difference. The output parameters describe the characteristics which symbolise the result of the recycling process, such as energy effort or process cost. This template enables a comparison of product variations concerning their recovery possibilities. The acquisition of data is described in the following.

\section{Information architecture}

The objective of the system is to integrate the partner's data base structure into the entire system. The amount of adaptation should be kept as small as possible and in the first step, it should be carried out by the system itself. Here, the difficulties are the different data base systems, the different data models as well as the various descriptions of similar aspects. These problems are separated in statically translations and model transformation.

Similar data base applications exchange their information according to a strictly prescribed pattern without a semantic check. Here, the objects, also called entities, of one system are transformed into the according entities of the other system. Simple examples for this procedure are CAD data conversions like IGES in STEP. In other cases, the entities of one model are transformed in another model's representation. This can include changes according to content, data format, file format and access type.

The viewed model combines the advantages of an integration platform with a complex transformation logic and supports the automatic adaptation of heterogeneous data base systems. The entire system consists of a central coordination system and single local systems (figure 7). The management and the coordination is placed in a central node where a predefined unified data meta model already exists.

The unified data meta model (UDMM) covers the structure of all information concerning the recycling processes, the recycling techniques and the product model. The unified model gives the possibility of transforming and including other models as well as the development of general valid queries ( Koonce et al 1996 ). The link manager is responsible for the registration and for the distribution of information from and to new partners or local data bases. In this indirect communication through the central management, the message board serves as a buffer for information or objects. Queries and selected data are kept in the board. 


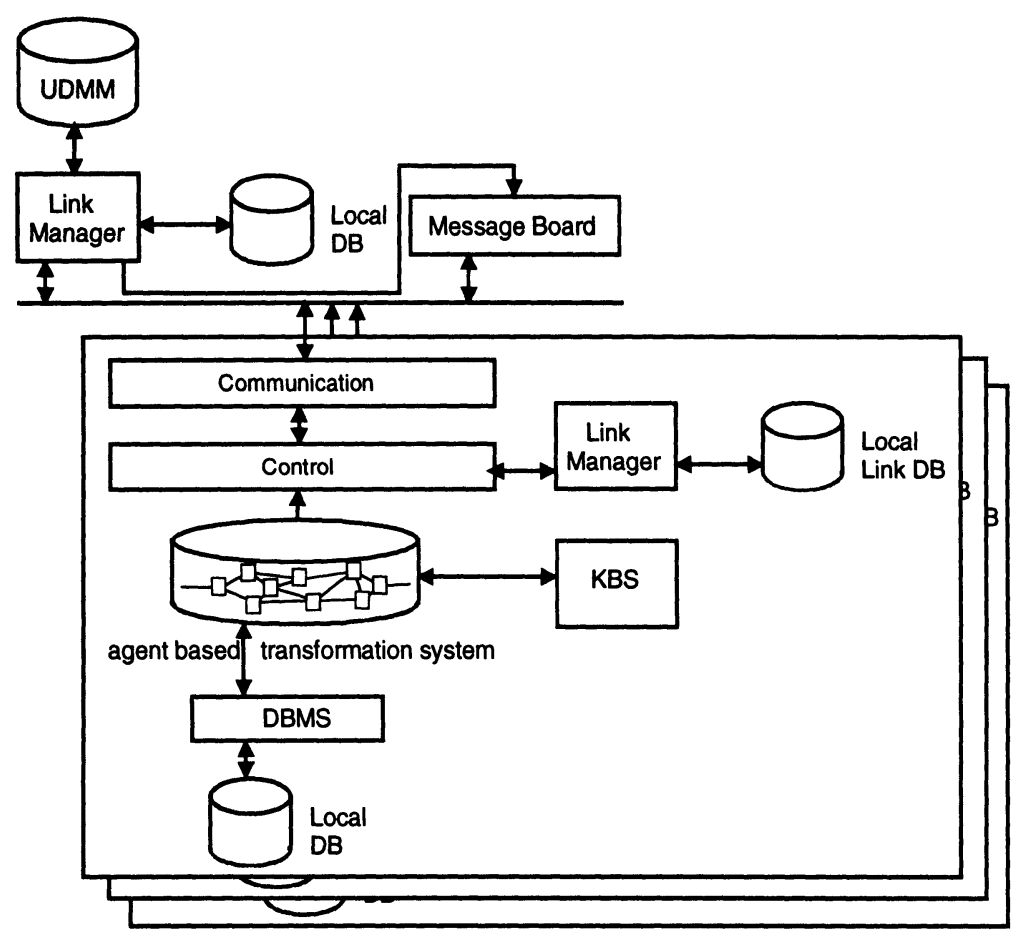

Figure 7 The federation principle

Connections which were enabled once are stored in the link data base. This enables a faster provision of information for new queries. As an example, the designer can be informed of all parameter changes that concern the recycling process.

The local data of the designer, the recycler and the developer initialise the federalised system. After the registration and configuration it is necessary to exchange information with other integrated systems.

The communication module serves as a connection to the network and to the central management system. The control module passes the incoming information to the agent-based transformation system.

The local link manager is responsible for establishing connections without using the central management. The local link data base stores the related information.

The transformation platform is an agent-based system (Mill 1995). It includes a variable-connected structure of transformation agents. Dependent on the incoming information the agents are self-activating. This leads exemplary to a transformation of multiple recycling process views into the information required by the designer. The Knowledge Based System (KBS) is connected to the agent based transformation system. It includes translating data, a transformation agent library and the transformation logic. The transformation logic incorporates the sequences of used agents. For instance mass percentages have to be changed into an absolute weight, or two input restrictions have to be transformed into a mixed relation. 
The local data base system contains private company data as well as a part of the federated data structure. It provides local access administration, data security and data organisation.

\section{SUMMARY AND OUTLOOK}

The described system supports the exchange of information between the designer, the recycler and the developer of recycling techniques. The designer is relieved from the search of process alternatives and he can quickly assess alternative recovery possibilities of products.

During product life cycle reviewing assessments prove the results. The producer can adapt his recycling-strategies to new influences. Recyclers can use existing facilities more effective and improve the recycling results. Developers of recycling techniques can test their developments and discover new application areas. The access to design data enables the simulation of new recycling procedures and equipment.

A federated data base system is used for the data administration. The system includes existing heterogeneous data bases owned by the companies. A unified data meta model is defined. The connection of the local data bases is user-friendly and automatically executed by an agent based transformation system.

The first development stage is the integration of the federation members and the search for information in their data bases. The second stage is the acquisition of information using information agents. The search within new information systems (data warehouses) via Internet is possible. Such data structures can now extend the unified data meta model. An object oriented architecture (CORBA) realises data access of several information media through a single application.

\section{REFERENCES}

Zussman, E., Kriwet, A., Seliger, G. (1994) Disassembly-Oriented Assessment Methodology to Support Design for Recycling. Annals of the CIRP Volume 43/1/1994. Berne, Stuttgart: Hallwag Publishers Ltd.

Nickel, W. (1996) Recycling-Handbuch, Strategien-Technologien-Produkte. VDIVerlag, Düsseldorf.

Löhr, K.; Melchiorre, M. (1995) Aufbereitungstechnik-Recycling von Produktionsabfällen und Altprodukten. Carl Hanser Verlag München Wien.

VDI-Richtlinie 2243 Blatt 1(1993) Konstruieren recyclinggerechter technischer Produkte, Grundlagen und Gestaltungsregeln. Beuth Verlag, Berlin.

Song, L., Nagi, R. (1995) An Integrated Information Framework for Agile Manufacturing. 5'th Industrial Engineering Research Conference Proceedings.

Dreer, P., Koonce, D. A. (1995) Development of an Integrated Information Model for Computer Integrated Manufacturing. Computers industrial Engineering Vol. 29, No. 1-4, Elsevier Sience Ltd. 
Orfali, R., Harkey, D, Edwards, J. (1996) The Essential Client/Server Survival Guide. John Wiley\&Sons Inc.

Koonce, D.A., Judd, R.P., Parks, C.M. (1996) Manufacturing Systems Engineering and Design : An intelligent Multi-Model, Integration Architecture. Computer Integrated Manufacturing Vol. 9, No. 6.

Mills, J. J. (1995) An Integrated Information Infrastructure for Agile Manufacturing. Manufacturing Science and Engineering ASME MH-Vol.3-2.

\section{BIOGRAPHY}

Prof. Dr.-Ing. Günther Seliger, born 1947, studied industrial engineering at the Technical University of Berlin and received his doctor's degree in 1983. After holding different positions at the Institute for Machine Tools and Factory Management (IWF) and at the Institute for Production Systems and Design Technology of the Fraunhofer Society he became a professor for assembly technology and factory management at the Technical University of Berlin in 1988. $\mathrm{He}$ is the vize president of the Technical University of Berlin and the spokesman of the special research program 281 „Disassembly Factories".

Dipl.-Ing. Thomas Keil, born 1972, studied information technology at the Technical University of Berlin. Since 1998 he is working as a research assistant at the IWF specialising in agile production systems and robotics.

Dipl.-Inform. Dirk Krützfeldt, born 1967, studied information technology at the Technical University of Berlin. Since 1995 he is working as a research assistant at IWF specialising in distributed production simulation.

Dipl.-Ing. Katrin Müller, born 1967, studied mechanical engineering with a focus on design technology at the Technical Universities of Magdeburg and Chemnitz. Subsequently, she worked as a project engineer. In 1995 she started working at the IWF as a research assistant specialising in methods of assessing recyclingsuitability.

Dipl.-Ing. Holger Perlewitz, born 1969, studied mechanical engineering with a focus on production technology at the Technical University of Berlin. Since 1996 he is working as a research assistant at the IWF specialising in computer aided disassembly planning and control. 